\title{
Preventing a Second Chernobyl
}

\section{-The Results of Efforts to Eliminate the Effects of Fukushima's Low-dose Radiation Contamination-}

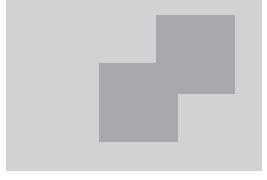

\author{
Louis Pasteur Center for Medical Research Foundation, \\ Kazuko Uno
}

\begin{abstract}
It has already been 3 months since I published the book "Overcoming Low-Dose Radiation" in late July, $2013^{1)}$. I am very happy with the feedback I have received such as, "The book made it easier to understand the lecture" or "It cheered me up" from people in Fukushima to whom I gave the book. Moreover, my senior colleagues, radiobiology and molecular biology professors at Kyoto University, commended me for the hard work undertaken to complete the book. The kind words from these professors emeritus, who are usually stern, were encouraging. In addition, I was surprised to receive emails and letters from people specializing in nuclear energy or material technology who agreed with the new perspectives in the book and offered their congratulations for its publication. I am surprised by the reaction to the book; I thought what I wrote was simple common knowledge in my field. This paper will discuss my intentions behind this book and what I learnt through the discussions with researchers in other fields while writing this book.
\end{abstract}

\section{Why were the Opinions of Scientists Divided?}

Looking back on 3.11, I was anxious for information about what to do while glued to the images of the earthquake, tsunami, and nuclear power plant accident. The biggest cause of concern was the risk of cancer stemming from radioactive substance from the Fukushima Daiichi Nuclear Power Plant. Moreover, many controversial public comments were rampant which exacerbated the fears in the affected areas. The general public, including scientists insisted that at the very least, children in Fukushima should be evacuated and those even in Tokyo should be moved to Kansai. As an immune researcher I believed that I could offer my knowledge to help ameliorate the fears regarding the risk of cancer. Many commentators on the Internet and television argued that mutant cells are created when humans are exposed to radiation. Since cells in the growth phase are more sensitive to radiation, children are over ten times more likely to develop cancer than adults. As such, these commentators predicted that one after another child in Fukushima would develop cancer within a few years. In a lecture on how to find motivation and meaning in life given to cancer patients I explained the mechanism of cancer and why radiation increases the risk of cancer. The lecture slides were

DOI : 10.15669/fukushimainsights.Vol.1.270

(C) 2021 Atomic Energy Society of Japan. All rights reserved.

Originally published in Journal of the Atomic Energy Society of Japan (ISSN 1882-2606), Vol. 56, No. 1, p. 15-18 (2014) in

Japanese. (Japanese version accepted: October 17, 2013) 
posted on the website of NPO Einstein, a volunteer group of scientist headed by Dr. Masako Bando, formerly the president of the Physics Society of Japan. My point of view is that the exaggeration of cancer risks on the Internet and in the media creates fear which weakens the immune system and increases the cancer risk. I believed I had a responsibility to speak out against it.

While radiation levels in Fukushima immediately after the nuclear power plant accident was indeed high, the consensus among medical/biological professionals around me was that the cancer risk of smoking tobacco was still higher than that associated with the radiation. In a mailing list distributed to many medical professionals, a doctor in Tokyo wrote that, "A patient of mine asked me to prescribe him sleeping pills for insomnia due to anxiety about radiation contamination. I wrote the prescription for him but was perplexed that he was quite concerned about radiation and yet he is a smoker" Indeed, one-third of cancer cases are caused by smoking. Another one third originates from dietary habits. Other causes include virus infection, work environment, and their living environment. The percentage of cancer cases attributed to radiation and ultraviolet rays is only about $2 \%$ of the total.

However, some of my colleagues who are physicists insist that it is inappropriate to consider the harm induced by tobacco and those by radiation as equivalent. It was difficult to convince them that tobacco and radiation both create oxygen radicals species (active oxygen species). I explained that a majority of DNA damage is caused by oxygen radicals that are generated when radiation breaks down water molecules. Oxygen radicals are created daily in an organism during respiration; it is used for bactericidal and other beneficial actions, however it also has harmful effects in the organism. Furthermore, I explained that living organisms have now developed a system for overcoming the harm related to oxygen radicals, and that most DNA damaged by radiation, ultraviolet ray, and breathing is repaired immediately. Thus, these physicists finally understood both radiation and smoking could be discussed in terms of oxygen radicals.

\section{Effects of Low-Dose Radiation and Ways to Overcome it}

\section{Cancer-Prevention Diet}

I believe that a diet that prevents cancer and adult diseases is effective to overcome the impact of low-dose radiation. Noteworthy examples of such diets include those presented in the McGovern Report and the Designer Foods Project. The McGovern Report published in 1977 in the U.S., stated that many chronic diseases originate from improper meat-based dietary habits and cannot be cured by drugs. The report pointed out that excessive consumption of fat, sugar, and salt are directly linked to life-threatening diseases such as heart disease, cancer, and stroke. The Designer Foods Project conducted by the National Cancer Institute in the U.S. researches plant-based compounds, such as phytochemicals, that they believe to be anti-carcinogenic. One of the aims of the project is to promote the consumption of these cancer-fighting foods that have phytochemicals and other compounds. Indeed, numerous compounds, which counters the harm inflicted by oxygen radicals, is contained in vegetables and fruits. Phytochemicals such as carotenoid, polyphenol, vitamin C, and vitamin E have particularly strong antioxidant effects that have been proven to aid in preventing cancer.

Fukushima Prefecture is an agricultural district that grows vegetables and fruits that are high in antioxidants. When I reflected upon the advice I can give to people of Fukushima, I came to the conclusion that "one should eat vegetables and fruits from Fukushima as much as 
possible when the measurement of their radiation dose shows no problem. Upon hearing this advice, a doctor from the Fukushima Japanese Red Cross Society commented that such a diet is quite similar to the adult-disease prevention diet that they were promoting. Indeed, not only does the antioxidant diet lead to cancer prevention, it also aids in preventing common adult diseases. Although Fukushima has a high rate of heart disease, it is possible that after ten years, residents in the prefecture can have a long life expectancy if salt intake is reduced and the anti-oxidant diet is implemented.

\section{Oxygen Radical is Keyword}

As was the case for my colleagues the physicist, the mechanisms of oxygen radicals and radiation can be understood once explained and can lead to a deeper mutual understanding of the topic. The influence of oxygen radicals is diverse. In the field of anti-aging medicine, oxygen radicals is reviled as the biggest cause of "rusting" or deterioration of the human body. It has been proven that oxygen radicals plays a role in many diseases that accompany aging, such as arteriosclerosis, myocardial infarction, Alzheimer's, cancer, diabetes, gastric ulcer, and cataracts. Recently, there is an abundance of supplements on the market designed to counteract oxygen radicals commonly referred to as free radicals. However, oxygen radicals should not be thought of as having only negative effects on an organism. The opposite is true; they are essential. For example, neutrophils and monocyte in white blood cells use oxygen radicals to kill bacteria, and this is one of their positive functions.

Biologists agree that when creatures started to live on land and breathe oxygen, they evolved to acquire a system to utilize this potentially dangerous oxygen radicals and overcome its harmful qualities. It is true that on a daily basis, organisms repair the damage inflicted by oxygen radicals that was produced by radiation, smoking, carcinogenic substances, or their own breathing. Based on this information, we can understand that radiation is not exceptional, but something that naturally occurs within our bodies and the environment.

\section{Natural Progression History of Cancer}

Cancers do not develop in overnight. Cells become carcinogenic only after several mutations. Moreover, it takes about 20 to 30 years for solid tumors to attain a clinically diagnosable size, with the exception of cancers such as leukemia or thyroid cancer. Cancer that is discovered one or two years after the nuclear power plant accident cannot be regarded as the consequence of the radiation from accident; it is more reasonable to think that the roots of such cancers were already present before the accident. After the nuclear plant accident, researchers who are not aware of the mechanism of cancer strongly predicted that 100,000 people living within a $100 \mathrm{~km}$ radius from Fukushima will have cancer in the next 10 years. These types of predictions caused unnecessary anxiety among many residents. However, this anxiety is detrimental, since the last defense against cancer cells in the human body is immune cells such as the natural killer cells. In fact, a vast majority of the mutant (carcinogenic) cells that humans produce daily is removed by natural killer cells and other immune cells. Fear, anxiety and other stressors reduce the function of these immune cells. Knowing this, I was concerned that exaggerated information about the effects of radiation would increase the risk of cancer in Fukushima residents due to increased stress. My hope was that my book would help to alleviate this anxiety, and offer a solution that would boost residents' immune system to mitigate the effects of stress and any exposure to low dose radiation. 


\section{Outreach Activities in Fukushima}

\section{1st Stage: 2011 Radiation Measurement by Japan Society for the Promotion of Science Volunteers}

Professor Ryuichi Shimizu of Osaka University, who attended my lecture in Kyoto in July invited me to Fukushima Projects. As a result, I joined and participated in a series of studygroup projects held between October to December, 2011 in Shirakawa city, Fukushima Prefecture. I participated as a member of the Industry-academia Cooperation Research Project of Japan Society for the Promotion of Science (JSPS) (commonly referred to as JSPS team for measuring and explain radiation). This is an outreach program organized by JSPS to assist Fukushima residents in overcoming the physical and emotional challenges they were facing due to the effects of the radiation. The outreach program was staffed mainly by volunteer researchers. JSPS team members measured radiation levels in the affected areas and offered explanations to residents. Shirakawa city created the study group so that researchers could give residents accurate information on how to guard their health from radiation. The meetings were held over three months, once in each of the eight districts of Shirakawa city from 6 p.m. to about 9 p.m. One of the main characteristics of our team was the fact that we walked around schools and other areas in the daytime before the meeting started and measured radiation levels. Radiation levels in the air measured between $0.1-0.7 \mu \mathrm{Sv} / \mathrm{h}$ and $0.2-0.5 \mu \mathrm{Sv} / \mathrm{h}$ on school playgrounds. This proved to us the effectiveness of decontamination, especially in school environments. (However, we noted that unlike schools in the cities, in schools surrounded by mountains, radiation levels increased slightly every time it rained). We were told that the contaminated soil taken from areas around the school was buried in the center of the school playground. It would be natural to think that the playground would have the lowest radiation levels and is safe, meaning that a common conclusion would be to let children spend as much time as they want on the playground. During the study-group lectures, the JSPS team reported the actual radiation levels around the city. On Google Map, displaying actual radiation levels and photographs of the areas where the measurements were taken such as an elementary school yard or hotspots under a drainpipe, made it easier for the residents to understand where actual elevated radiation spots were in their neighborhood. These lectures created a forum where residents could calmly express their concerns and receive factual feedback; this outreach program lead to our team receiving a certificate of appreciation from Shirakawa City.

\section{2nd Stage: 2012 Relaxation and Antioxidant Experiment}

Mothers with small children stood to benefit the most from the study groups. However, since the meetings were held in the evenings it was difficult for mothers to attend. This led me to think that it was necessary to have childcare available at the meetings. However, when I requested support this project to a female member of Fukushima prefectural assembly in April, she replied, "mothers in Fukushima are extremely stressed at the moment, so aromatherapy would be more welcomed than lectures by famous scientists." This reaction prompted me to introduce cosmetic therapy to the women in Fukushima. Using a sample of aged women, I had already demonstrated to the effectiveness of cosmetic therapy in activating natural killer cells and IFN- $\alpha$ production which are the index of a immune activity ${ }^{2}$. JSPS was a little reluctant to undertake the project. However, I was invited to a lecture organized by the Japanese Red Cross Society, and at the time, I proposed adding cosmetic therapy sessions to 
the study group in Fukushima. The team at Red Cross was already aware of the effectiveness of spa therapies such as footbaths and aromatherapy relaxation, so they agreed, saying they were onboard with anything that helps the disaster victims. Additional support for the cosmetic therapy project came from Naris Cosmetics, a company that had supported my prior cosmetic therapy research. Naris Cosmetics is located in Fukushima Ward, Osaka, and they assisted me with this project partly because their headquarters is located in Osaka, in a city that shares the same name as Fukushima. The company generously donated facial lotions and other cosmetics for 10,000 participants and this allowed the launch of the cosmetic therapy project. Hand massages with Naris lotions were offered during the study meetings and during these massage sessions, volunteers answering questions and offering more information helped the female participants to deepen their understanding of the lectures. I believe that this spa project helped to put not only the females but also males in the community at ease and so it was particularly unique to female researchers.

\section{3rd Stage: 2013 Training Peer Educators}

I believed that simply explaining that an appropriate cancer fighting diet is equivalent to generous amounts of fruits and vegetables high in antioxidants was not enough to convince Fukushima residents to change their eating habits. As a result, during the autumn of 2012, I introduced an experiment to demonstrate the antioxidant capacity of fresh fruits and vegetables to residents. It was a simple experiment wherein the juice of a vegetable or fruit was extracted and mixed with diluted isodine solution (it can be used as antiseptic solution and also for oxidation-reduction titration) to observe the change in color. It was easy to see the anti-oxidative effect: the brown isodine solution immediately turned transparent when green tea, garlic, or green pepper juice was added. The mothers in the study group were positively influenced by the experiment, and there was one mother who told her child to eat green peppers from now on.

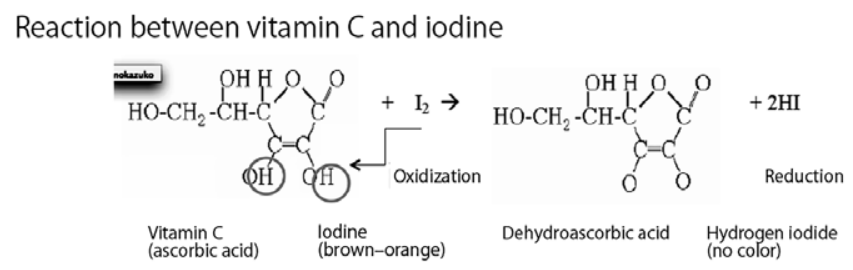

A representative of a group of affected residents who evacuated from Fukushima to Kyoto, visited NPO Einstein on June 2011. She requested our help for these evacuees to receive whole body counter test to determine their radiation levels and/or tests to check their thyroid function.

Whole body tests are performed free of cost in Fukushima by a mobile testing center housed in van. These evacuees were denied testing service because people who were physically in Fukushima were given priority and this mobile testing center could not come to Kansai Region anytime soon. We at NPO Einstein discovered that Kyoto University Research Reactor and Mihama Nuclear Power Plant have a whole body counter, and with their support, we visited both institutions with the Fukushima evacuees. Before undergoing the test, we had an orientation session in the chartered bus to explain the whole body counter, what the equipment measures, and how the results of the whole body counter in Fukushima fared. 
In addition, we also asked the staff from these institutions to give a talk. In addition to Einstein researchers, evacuees who already underwent radiation testing participated, and spoke about their experience to the evacuees who were being tested for the first time. Through this experience, we came to understand that people who were anxious about doing their amount of exposure got more comfort from explanations given by a fellow evacuee than explanations provided by researchers.

In 2012, I was requested by the Fukushima local government to hold several health workshops for public health workers and again in 2013 for both public health workers and public schools teachers. The primary goal was to enable workshop participants to interact with local residents with confidence. There are many Fukushima residents who are still anxious and even though I visit every month, there is a limit to the number of people I can council at any given time. My hope is that those fortunate to attend my lectures will confidently relay information to the people around them, and in this way, residents' concerns will slowly fade and be replaced with more optimistic thinking.

\section{Crisis Communication}

\section{The Power of Network}

I believe that one of the main issues underlying the inadequate response to the needs of Fukushima residents during this crisis was the poor communication of accurate, relevant information on the part of scientists. Personally, as a scientist, I was particularly disappointed in the barrage of misinformation surrounding the biological impact of low-dose radiation that was communicated during the early months after the accident. Researchers who attempted to reassure the public that at the current level there was no need to worry about radiation effects were accused of taking sides with the government. Several hackneyed theories about the impact of radiation were thrown around; these theories were based on experiments conducted in test tubes only, data that were several decades old, and exaggerated case reports. Those who sided with the opinion that "danger" existed tended to be recognized as being conscientious. During this time, I was attempting to verify the many theories about radiation that were circulating and trying to connect the theories with their original sources. We concluded that most of these exaggerated warnings were because: (1) mere sections of an entire paper were taken out of context and the risks magnified, (2) the original source were merely case reports that would not qualify for peer review and could hardly be considered as reliable academic publications, and (3) the results published in a single paper on epidemiological studies were likely treated as being typical example for the entire subject. We recognized that it was necessary to uncover if they were more reliable papers including those from other countries that reported similar impact.

As such, we created a discussion forum at NPO Einstein that included researchers from various fields including physics, radiobiology, medical science, and nuclear engineering.

\section{Role of Scientists}

Rapid, cutting-edge advances in the field of science has created the need for a new profession called scientific communicators who can relay complex scientific information in a way the general public can understand. However, these scientific communicators made very little worthwhile contributions during this crisis. As far as I know, they either exaggerated the 
impact of low-dose radiation based on their rather limited knowledge and caused confusion, or simply stayed silent. Meanwhile, I thought that even though there was very little support from these communication specialists from various fields, some citizens who are avid critical thinkers were able to better assess the situation and make more accurate judgments. One could find such people among the evacuees from Fukushima. The difference between these people and the science communicators is that the evacuees had a strong connected to, and history with Fukushima

During the disaster recovery period, retired researchers played a particularly important role. Many active researchers, due to their work obligations, either visited Fukushima very few times, or not at all. Additionally, they tended to have a narrower perspective compared to the older retirees who have the benefit of years of experience in their respective discipline. To overcome the dire situation in Fukushima, it is perhaps more effective to invest in programs in which retired researchers train peer educators (people who guide the sharing and propagation of appropriate knowledge/skill/action in their community) instead of training science communicators. In the field of AIDS study for example, the investment in developing peer educators is showing some results ${ }^{3)}$.

\section{Chernobyl vs. Fukushima}

An onslaught of books about Chernobyl was published after the Fukushima Accident, many discussing how children from Chernobyl are inflicted with many diseases. Reading these books would make an anxious person even more concerned. I found the methods to reduce radiation in vegetable and meat that was mentioned in the book "How to Protect you and your Children from Radiation" especially problematic, as these suggestions were meaningless unless the actions were taken immediately after the accident. More worrisome, is that the advice presented could also have negative health effects by decreasing the nutrients and microelements in these natural foods. Although Japan should learn from Chernobyl's system of testing the safety of their food chain, such a system is already in place in Fukushima, and instruments to measure radiation levels in locally grown produce and animal products are installed in local community centers and other locations. As such testing is not available in other prefectures; I expect that in the future, foods from Fukushima will be regarded as safer because they have been more carefully tested.

I am of the opinion that Fukushima will not be a second Chernobyl for the following reasons:

(1) The estimated radiation dose detected after the Fukushima Daiichi Nuclear Power Plant Accident was about 1/10th that of the Chernobyl Accident.

(2) In Fukushima, the distribution of milk contaminated by iodine was halted quite quickly after the accident.

(3) Unlike residents of other regions who are prone to have chronic iodine deficiency, Japanese tend to naturally consume healthy amounts of iodine in their diets through seaweed and other foods.

(4) From the results from Co-op Fukushima extra-meal research (households prepared an extra single serving for every meal the family ate over a two-day period; these meals were evenly rotated and their radiation levels were measured), we know that as long as residents rotate the food they consume, there is little need to worry about the accumulation of radioactive materials from foods.

(5) Unlike the areas around Chernobyl where it was difficult to obtain produce high in 
antioxidants in winter, these fruits and vegetables can be obtained far more readily year round in Japan.

(6) Published reports showed that the soil in areas affected by the Chernobyl Accident such as in Belarus and the prairie in Ukraine, were low on minerals, making them even more susceptible to contamination by radioactive materials ${ }^{4)}$. Moreover, while the addition of land-improvement agents succeeded in reducing the contamination level of agricultural produce, these agents also tend to reduce the absorption of microelements necessary to support healthy soil organisms, such as cobalt, zinc, copper, iron, manganese, and potassium. On the contrary, the soil composition in Fukushima is significantly different from in the Ukraine, and the food chain in Fukushima did not have the levels of radiation contamination that occurred in Chernobyl and the surrounding regions.

(7) In Fukushima, whole body counter did not detect a problematic amount of cesium in most people. However, in Chernobyl, 20-70 Bq/kg radiation doses are still being detected in many residents even 10-20 years after the accident.

\section{References}

1) Uno K. Overcoming Low-Dose Radiation Contamination. Shogakukan Shinsho; 2013. [in Japanese]

2) Uno K, Tani M, et al. Effect of Cosmetic Therapy on Old Women. Nihon=Sei-Kenkyukaigi Kaiho (J Japan = Sexuality Study Council). 2001; Issue 13: 11-19. [in Japanese]

3) Takamura S. Peer Counseling Manual to Support the Sexual Health of Puberty. Shogakukan; 2005. [in Japanese]

4) Shiraishi K. Radiation and Nutrition. Miyaobi Publishing; 2011. [in Japanese] 